\title{
Hypoxic preconditioning increases the protective effect of bone marrow mesenchymal stem cells on spinal cord ischemia/reperfusion injury
}

\author{
ZHILIN WANG, BO FANG, ZHIBIN TAN, DONG ZHANG and HONG MA \\ Department of Anesthesiology, The First Affiliated Hospital of China Medical University, \\ Shenyang, Liaoning 110001, P.R. China
}

Received October 27, 2014; Accepted July 23, 2015

DOI: $10.3892 / \mathrm{mmr} .2016 .4753$

\begin{abstract}
Transplantation of bone marrow mesenchymal stem cells (BMSCs) protect against spinal cord ischemia/reperfusion injury (SCIRI). However, a large number of transplanted BMSCs often undergo apoptosis, which severely affects the treatment outcome. Previous studies have demonstrated that hypoxic preconditioning effectively increases the survival rate of BMSCs following transplantation, and increases their protective effect on injured tissues. However, there have been few reports regarding roles of hypoxic preconditioning in SCIRI. The present study isolated rat BMSCs and separately transplanted hypoxia- and non-hypoxia-preconditioned BMSCs into the spinal cord tissues of rats with SCIRI. The role of hypoxic preconditioning in the promotion of the protective effect of BMSCs on SCIRI was investigated using neurological function scores, Evans blue staining, hematoxylin and eosin staining and terminal deoxynucleotidyl transferase dUTP nick end labeling. In addition, reverse transcription-quantitative polymerase chain reaction and western blotting were used to detect the expression levels of hypoxia-inducible factor $1 \alpha(\mathrm{HIF}-1 \alpha)$, and to investigate its possible underlying mechanism of action. The results indicated that hypoxic preconditioning effectively increased the protective effects of BMSCs on neurological function, blood spinal cord barrier and tissue damage following SCIRI, and inhibited apoptosis. Furthermore, hypoxic preconditioned BMSCs upregulated the expression of HIF-1 $\alpha$ in spinal cord tissues. Therefore, hypoxic preconditioning effectively increased the protective effect of BMSCs on SCIRI and may be associated with upregulation of the expression of HIF-1 $\alpha$. Hypoxic preconditioning may
\end{abstract}

Correspondence to: Dr Hong Ma, Department of Anesthesiology, The First Affiliated Hospital of China Medical University, 155 North Nanjing Street, Shenyang, Liaoning 110001, P.R. China E-mail: hongmasy@163.com

Key words: spinal cord ischemia/reperfusion injury, bone marrow mesenchymal stem cells, hypoxic preconditioning, hypoxia-inducible factor $1 \alpha$ serve as an effective means of increasing the protective effect of BMSCs on SCIRI.

\section{Introduction}

Spinal cord ischemia/reperfusion injury (SCIRI) refers to the return of the blood supply in recovery following trauma of the spinal cord. The neurological function of the spinal cord cannot be improved, and the injury is further aggravated by ischemia, which leads to the irreversible, delayed death of spinal neurons (1). SCIRI is a common complication following thoracic or thoracoabdominal aortic surgery (2). Previous studies reported that the incidence of spinal cord injury caused by ischemia/reperfusion is 3-18\% (3), and patients with SCIRI have a poor prognosis, usually resulting in severe paralysis or mortality $(4,5)$, which places burdens on patients and family members. Therefore, the identification of effective preventative measures is important for improving the prognosis of SCIRI.

Previous studies have demonstrated that bone marrow mesenchymal stem cell (BMSC) transplantation effectively attenuates SCIRI (6-8). However, high levels of apoptosis in ischemic tissues following cell transplantation severely affect treatment outcomes. Studies have reported that the survival rate of autologous transplanted cells in myocardial ischemia patients was only $1 \%$ (9), and although the survival rate of BMSCs transplanted into ischemic myocardial tissues on the first day was $>99 \%$, the survival rate on the fourth day decreased sharply to $<0.44 \%$ (10). Although the cause of transplanted cell death remains to be elucidated, insufficient blood supply, hypoxia, inflammation, oxidative stress and accumulation of cytotoxic substances all have adverse effects on the survival of transplanted cells (11). To date, several in vitro and in vivo studies have determined that hypoxic preconditioning may increase the adaptability of mesenchymal stem cells to hypoxic environments, increase cell activity and inhibit apoptosis (12-16). However, the function of hypoxic preconditioning on the protective effect of BMSCs in SCIRI have not been reported.

In the present study, the effect of hypoxic preconditioning on the protective role of BMSCs in SCIRI was explored. Hypoxic preconditioning is involved in improving the survival of BMSCs and promoting the recovery of SCIRI. Hypoxic 
preconditioning may become a promising adjuvant therapy in the treatment of SCIRI with BMSCs.

\section{Materials and methods}

Experimental animals. A total of 30 eight week-old male healthy adult Sprague-Dawley (SD) rats weighing 200-250 g were purchased from the Experimental Animal Center of the China Medical University (Shenyang, China). Prior to experimentation, the rats were housed separately at $22^{\circ} \mathrm{C}$ with $40-50 \%$ humidity and a $12 \mathrm{~h}$ light/dark cycle in animal rooms for adaptive feeding for 1 week. The rats had access to food and water ad libitum. The care and treatment of the animals and present study was approved by the Experimental Animal Ethics Committee of the China Medical University.

Isolation, culture, identification and hypoxic preconditioning of rat BMSCs. According to previously described methods $(17,18)$, the rats were sacrificed by cervical dislocation, and the femur and tibia were removed under sterile conditions. The bone marrow cavity was exposed, and Dulbecco's modified Eagle's medium (DMEM; Gibco Life Technologies, Carlsbad, CA, USA) supplemented with $10 \%$ fetal bovine serum (FBS; GE Healthcare Life Sciences, Logan, UT, USA) in a $2 \mathrm{ml}$ syringe was used to wash out the bone marrow. Following centrifugation at $252 \mathrm{x} g$ for $10 \mathrm{~min}$ at room temperature, the supernatant was discarded. The cells were resuspended by red cell lysis buffer (Beijing Solarbio Science \& Technology Co., Ltd., Beijing, China) and centrifuged at $112 \mathrm{x}$ g for $10 \mathrm{~min}$ at room temperature. The cells were then filtered with a 200 mesh sieve and cultured in an incubator at $37^{\circ} \mathrm{C}$ in an atmosphere containing $5 \% \mathrm{CO}_{2}$ for $24 \mathrm{~h}$, prior to being inoculated at a density of $5 \times 10^{5} /$ flask. After $24 \mathrm{~h}$, the culture medium was replaced to remove the non-attached cells, and was replaced again after 5 days. Cells with optimal growth at the third generation were used to identify the expression levels of CD29, CD44, CD45 and CD90 using FACSCalibur (BD Biosciences, Franklin Lakes, NJ, USA). Hypoxic preconditioning of the cells was performed by culturing the cells in serum-free DMEM in a cell incubator $\left(37^{\circ} \mathrm{C} ; 3 \% \mathrm{O}_{2}, 5 \% \mathrm{CO}_{2}\right.$ and $\left.92 \% \mathrm{~N}_{2}\right)$ for $24 \mathrm{~h}$, as previously described by Liu et al (15). The cells were placed in DMEM in the absence of FBS and cultured at $37^{\circ} \mathrm{C}$ in an atmosphere containing $3 \% \mathrm{O}_{2}, 5 \% \mathrm{CO}_{2}$ and $92 \% \mathrm{~N}_{2}$ for $24 \mathrm{~h}$.

Experimental grouping. The SD rats were randomly divided into five groups, with six rats per group. The sham group received simple surgical manipulation without ischemia/reperfusion treatment; the SPIRI group (IR group) received SCIRI surgery; the non-preconditioned BMSC transplantation group (NP-MSC group) was injected with untreated BMSCs into the spinal cord sheath prior to ischemia/reperfusion; the hypoxic preconditioned BMSC transplantation group (HP-MSC group) was injected with hypoxic preconditioned BMSCs prior to ischemia/reperfusion; and the phosphate-buffered saline (PBS) group served as a control group. The PBS group received injection of an equal volume of PBS prior to ischemia/reperfusion. Based on a method described by Fang et al (8), cell transplantation was performed 2 days prior to SCIRI. A $10 \mu \mathrm{l}$ polyethylene tube was inserted into the subarachnoid cavity using a 16-gauge needle, and slowly moved to the cerebrospinal fluid (CSF). A total of $5 \times 10^{5} / 5 \mu 1$ cells or an equal volume of PBS was injected into the CSF, and the tube was removed. The heads of the experimental animals faced upwards for $60 \mathrm{~min}$, and the rats with normal fore and hind limb function were included in the present study.

Establishment of an SCIRI rat model. An aortic cross-clamping method was used to establish an SCIRI model in the rats, as previously described (19). Prior to experimentation, the rats were fasted (no food or water) for $6 \mathrm{~h}$ and anesthetized using 3\% halothane (China Sinopharm international Co., Ltd., Shanghai, China). Body temperature was monitored using DT-K101A rectal thermometers (Hangzhou Hua'an Medical \& Health Instruments Co., Ltd., Hangzhou, China) and during the experimental process, the body temperature was maintained at $37.5 \pm 0.5^{\circ} \mathrm{C}$ using electric blankets and heating lamps. The rats were placed in a supine position, and a longitudinal incision was made along the left vertex of the sternum to the second rib bone. The left superior vena cava and internal mammary artery were carefully avoided. The aortic arch was separated from the left common carotid and subclavian arteries, and the aortic arch and left subclavian artery were clamped at the same time using micro-artery clamps (Jinzhong, Shanghai, China). The artery clamps were opened after $10 \mathrm{~min}$, and the surgical wound was sutured layer by layer with 3-0 sutures (Shanghai Xincheng Medical Instruments Co., Ltd., Shanghai, China). An intraperitoneal injection of $5 \mathrm{ml}$ of $0.9 \%$ saline was performed following surgery, and the rats were placed in $28^{\circ} \mathrm{C}$ incubators for $3 \mathrm{~h}$ prior to being returned to their cages.

Neurological function scoring. Neurological function scoring in each group was performed at 6, 12, 24, $48 \mathrm{~h}$ and 7 days following reperfusion using the Tarlov criteria $(8,20,21)$. The scoring criteria were as follows: 0 points for the absence of evident hind limb motor function; 1 point for perceivable autonomic joint movement; 2 points for free movement of hind limbs, but an inability to stand; 3 points for an ability to stand but an inability to walk; and 4 points for total recovery of hind limb function and the ability to walk.

Detection of the integrity of the blood spinal cord barrier $(B S C B)$. A total of $2 \%$ Evans blue (Sigma-Aldrich, St. Louis, MO, USA) staining solution $(2 \mathrm{ml} / \mathrm{kg})$ was slowly injected into the tail vein of the rats. After $1 \mathrm{~h}$, the rats were anesthetized using $3 \%$ halothane, and infusion needles were inserted through the ascending aorta to infuse $500 \mathrm{ml} / \mathrm{kg}$ saline. Spinal cord tissue samples of the L4-L6 were removed and sectioned transversely into two parts. Following weighing, a section of the spinal cord tissues of $\sim 100 \mathrm{mg}$ was mixed with $1 \mathrm{ml}$ formamide (Amresco, LLC, Solon, OH, USA) and then immersed in $37^{\circ} \mathrm{C}$ prior to being centrifuged at $260 \mathrm{x} \mathrm{g}$ for $10 \mathrm{~min}$ at room temperature in order to collect the supernatant, and the absorbance was measured at $632 \mathrm{~nm}$ using a ELX-800 microplate reader (Bio-Tek Instruments, Inc., Winooski, VT, USA). Simultaneously, different concentrations $(0.05,0.1,0.2,0.4,0.8,1.6$, and $3.2 \mu \mathrm{g} / \mathrm{ml})$ of Evans blue solution were prepared, and a standard curve was plotted to calculate the concentration of Evans blue in each spinal cord tissue sample. The other section of spinal cord tissue was 
fixed in $10 \%$ paraformaldehyde (China Sinopharm international Co., Ltd.) to prepare $10 \mu \mathrm{m}$ sections. The stained tissue samples were observed under an IX53 fluorescence microscope (Olympus Corporation, Tokyo, Japan) and images were captured.

Hematoxylin and eosin (HE) staining. The injured rat spinal cord tissue samples from each group were fixed in $10 \%$ formaldehyde (China Sinopharm international Co., Ltd.) overnight and embedded in paraffin (Shanghai Hushi Laboratorial Equipment Co., Ltd, Shanghai, China) in order to obtain $5 \mu \mathrm{m}$ thick sections. HE staining (Beijing Solarbio Science \& Technology Co., Ltd.) was performed, according to standard procedures. Following staining, the slides were observed under a DP73 light microscope (Olympus Corporation) and images were captured.

Detection of apoptosis using terminal deoxynucleotidyl transferase dUTP nick end labeling (TUNEL). The levels of apoptosis were detected using an In Situ Cell Death Detection kit (Roche Diagnostics GmbH, Mannheim, Germany), according to the manufacturer's instructions. Paraffin sections were inactivated using $\mathrm{H}_{2} \mathrm{O}_{2}$ solution, prior to incubation with $50 \mu 1$ TUNEL reaction solution (Roche Diagnostics $\mathrm{GmbH}$ ) at $37^{\circ} \mathrm{C}$ for $60 \mathrm{~min}$. Following washing with PBS, the sections were incubated with $50 \mu 1$ converter-POD working solution (Roche Diagnostics $\mathrm{GmbH}$ ) at $37^{\circ} \mathrm{C}$ for $30 \mathrm{~min}$. Following development with $3^{\prime}$-diaminobenzidine (Beijing Solarbio Science \& Technology Co., Ltd.), the nuclei were stained with hematoxylin. The sections were observed under a microscope and the images were captured.

Reverse transcription-quantitative polymerase chain reaction (RT-qPCR). Total RNA was extracted using TRIzol ${ }^{\circledR}$ reagent (Invitrogen Life Technologies, Carlsbad, CA, USA), and RNA was reverse transcribed into cDNA using a cDNA First Strand Synthesis Reagent kit (Takara Biotechnology Co., Ltd., Dalian, China), according to the manufacturer's instructions. The sense and antisense hypoxia-inducible factor $1 \alpha$ (HIF-1 $\alpha)$ primers (synthesized by Sangon Biotech Co., Ltd., Shanghai, China) were 5'-CTCCCATACAAGGCAGCAGAAAC-3', and 5'-AGAAACGAAACCCCACAGACAAC-3', respectively, and the sense and antisense primers of $\beta$-actin were 5'-GGAGATTACTGCCCTGGCTCCTAGC-3', and 5'-GGCCGGACTCATCGTACTCCTGCTT-3', respectively. Quantitative analysis was performed using an Exicycler ${ }^{\mathrm{TM}} 96$ Quantitative Fluorescence analyzer (Bioneer Corporation, Daejeon, Korea). The total PCR reaction volume was $20 \mu \mathrm{l}$ and consisted of $1 \mu \mathrm{l} \mathrm{cDNA}, 0.5 \mu \mathrm{l}$ of each primer, $10 \mu \mathrm{l} \mathrm{SYBR}{ }^{\circledR}$ GREEN master mix (BioTeke Corporation, Beijing, China) and $8 \mu \mathrm{ldd} \mathrm{d}_{2} \mathrm{O}$. The PCR reaction thermocycling conditions were as follows: $95^{\circ} \mathrm{C}$ for $10 \mathrm{~min} ; 40$ cycles of $95^{\circ} \mathrm{C}$ for $10 \mathrm{sec}$, $58^{\circ} \mathrm{C}$ for $20 \mathrm{sec}, 72^{\circ} \mathrm{C}$ for $30 \mathrm{sec}$ and $4^{\circ} \mathrm{C}$ for $5 \mathrm{~min}$. The relative mRNA expression levels were calculated using the $2^{-\Delta \Delta C t}$ method (22).

Western blotting. Following tissue homogenization (S10; Scientz, Ningbo, China), total protein from the spinal cord tissue samples of each group was extracted using radioimmunoprecipitation assay lysis buffer (Beyotime Institute of
Biotechnology, Haimen, China) and centrifuged 10,010 x g for $10 \mathrm{~min}$ at $4^{\circ} \mathrm{C}$, and the protein concentrations were determined using a bicinchoninic acid assay (Beyotime Institute of Biotechnology). Total protein (40 $\mu \mathrm{g})$ from each group was subjected to $8 \%$ SDS-PAGE. Following electrophoresis, the proteins were transferred onto a polyvinylidene difluoride membrane (EMD Millipore, Billerica, MA, USA). The membrane was blocked with $5 \%$ non-fat milk at room temperature for $1 \mathrm{~h}$, and then incubated with polyclonal rabbit anti-rat HIF-1 $\alpha$ primary antibody (1:400; cat. no. BA0912-2; Wuhan Boster Biological Technology, Ltd., Wuhan, China) at $4^{\circ} \mathrm{C}$ overnight, followed by incubation with polyclonal goat anti-rabbit horseradish peroxidase-conjugated secondary antibody (1:5,000; cat. no. A0208; Beyotime Institute of Biotechnology) at room temperature for $45 \mathrm{~min}$. The development of the luminescent substrate was measured using an enhanced chemiluminescence kit (Shanghai 7Sea Pharmatech Co., Ltd., Shanghai, China). Following exposure, images were captured and scanned into a computer, and Image J 2.1 software (National Institutes of Health, Bethesda, MA, USA) was used to analyze gray density. The protein expression levels were quantified using $\beta$-actin as an internal control.

Statistical analysis. The data are presented as the mean \pm standard deviation. The comparison between groups was performed using one-way analysis of variance and multiple comparisons were performed using the Bonferroni post-hoc test. The processing of the data and figures was performed using Graphpad Prism 5.0 (GraphPad Software, Inc., La Jolla, CA, USA). P<0.05 was considered to indicate a statistically significant difference.

\section{Results}

Isolation, culture and identification of rat BMSCs. Rat BMSCs were isolated using density gradient centrifugation. Third generation BMSCs were harvested to perform an identification of the surface markers using flow cytometry (Fig. 1). The expression of CD29, CD44 and CD90 were positive, and the expression of CD45 was negative. These results were concordant with those of a previous study (23), indicating that rat BMSCs had been obtained successfully.

Hypoxic preconditioning increases the protective effects of BMSCs on neurological function. The neurological function score of the rats was determined in each group 6, 12, 24, $48 \mathrm{~h}$ and 7 days following SCIRI. The average score of each group is shown in Fig. 2. The hypoxic preconditioned and the untreated BMSCs promoted the recovery of neurological function following SCIRI; however, the neurological function in the HP-MSC group was significantly higher, compared with that of the NP-MSC group. From $12 \mathrm{~h}$ post-ischemia/reperfusion, the neurological function score in the HP-MSC group was already significantly higher, compared with that of the IR group $(\mathrm{P}<0.05)$; however, the neurological function of the NP-MSC and IR groups were not significantly different, indicating that hypoxic preconditioning increased the protective effect of BMSCs on neurological function.

Hypoxic preconditioning increases the protective effects of BMSCs on the BSCB. Evans blue staining was used to 

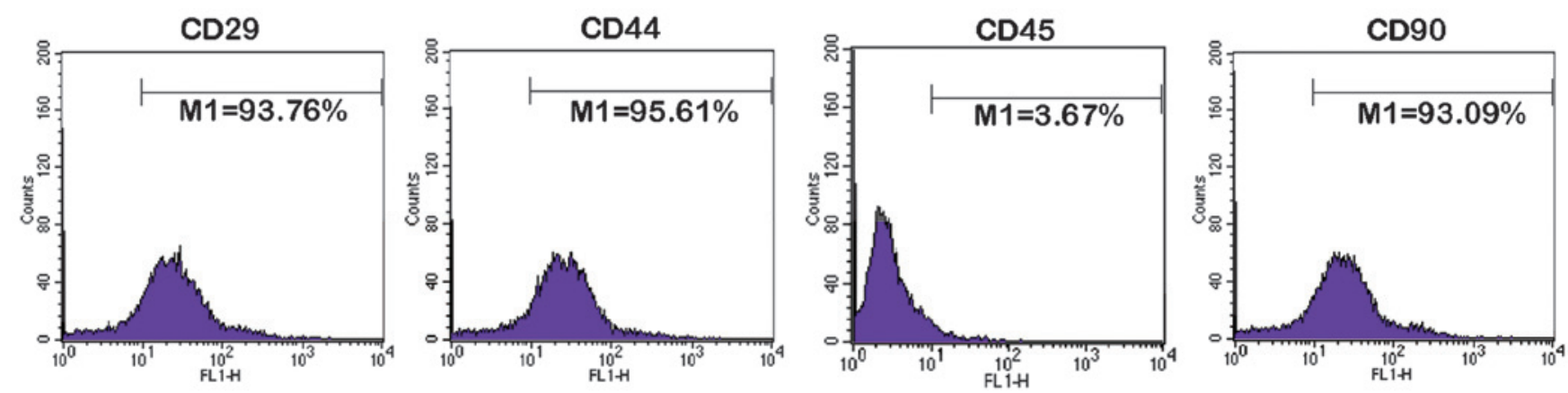

Figure 1. Identification of rat BMSCs using flow cytometry. BMSCs passaged to the third generation were identified using flow cytometry. The expression of CD29, CD44 and CD90 were positive, whereas the expression of CD45 was negative. BMSC, bone marrow mesenchymal stem cell. M1, percentage of cells with a fluorescence intensity $>10$.

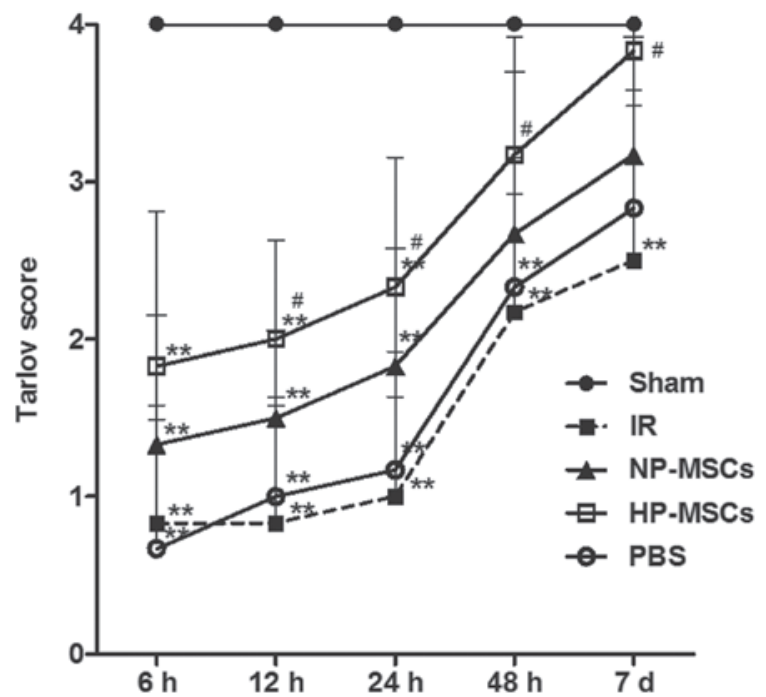

Figure 2. Neurological function scoring using the Tarlov method. At 6, 12, 24, 48 and 7 days following ischemia/reperfusion, the neurological function of the rats in each group was scored. Each group contained six rats and the data are presented as the mean \pm standard deviation. ${ }^{* *} \mathrm{P}<0.01$, vs. sham group; ${ }^{\#} \mathrm{P}<0.05$, vs. IR group. BMSC, bone marrow mesenchymal stem cell; Sham, sham-operated; IR, ischemia/reperfusion; NP-MSC, non-preconditioned BMSC transplantation; HP-MSC; hypoxic preconditioned BMSC transplantation; PBS, phosphate-buffered saline-treated.

investigate the protective effects of the treatments of each group on the BSCB following SCIRI (Fig. 3). The BSCB was severely damaged in the IR group, and significant Evans blue leakage (indicated in red) was observed under a fluorescence microscope. The Evans blue leakage rates in the NP-MSC and HP-MSC groups significantly weakened. The Evans blue concentration levels were consistent with the results of the fluorescence microscopy observations. The Evans blue concentrations in the damaged spinal cord tissue samples in the IR and PBS groups were significantly higher, compared with those of the sham group $(\mathrm{P}<0.01)$. Compared with the IR group, the Evans blue concentrations in the NP-MSC and HP-MSC groups were significantly decreased $(\mathrm{P}<0.05$ and $\mathrm{P}<0.01$, respectively) and those of the HP-MSC group were significantly lower, compared with those of the NP-MSC group $(\mathrm{P}<0.01)$. These results indicated that transplantation of BMSCs provided a certain protective effect on the BSCB, and that hypoxic preconditioning improved this function.
Hypoxic preconditioning increases the protective effects of BMSCs on spinal cord tissue injury. HE staining was performed on the tissue samples from each group to observe the effects of the various treatments on spinal cord injury (Fig. 4). Tissue injury in the IR and PBS groups was more severe, with nuclei fragmentation, formation of a large number of vacuoles and tissue bleeding accompanied by inflammatory cell infiltration. The NP-MSC and HP-MSC groups had fewer vacuoles and markedly decreased degrees of injury, and the tissue morphology of the HP-MSC group was similar to that of normal spinal cord tissues. Detection of the apoptotic levels in the spinal cord tissue samples using a TUNEL assay (Fig. 5) determined that an increased number of apoptotic cells were present in the IR and PBS groups, compared with the sham group, whereas the number of apoptotic cells in the NP-MSC group was decreased. The HP-MSC group exhibited apoptotic cells, however, their number was significantly lower than that in the NP-MSC group. These results suggested that hypoxic preconditioning increased the protective effect of the BMSCs on SCIRI.

Hypoxic preconditioning upregulates the expression of $H I F-1 \alpha$ and increases the protective effects of BMSCs on SCIRI. To investigate the possible mechanisms underlying the increased protective effects of BMSCs on spinal cord injury, the expression levels of HIF-1 $\alpha$ in the spinal cord tissue samples of each group were determined. Compared with the IR group, the mRNA expression levels of HIF-1 $\alpha$ in the NP-MSC and HP-MSC groups were markedly increased, and the mRNA expression of HIF- $1 \alpha$ in the HP-MSC group was significantly higher, compared with that in the NP-MSC group (Fig. 6A). The protein expression levels of HIF-1 $\alpha$ in each group were similar to the mRNA expression levels of HIF-1 $\alpha$ (Fig. 6B). Compared with the IR group, the protein expression of HIF-1 $\alpha$ in the NP-MSC group increased, while that of the HP-MSC group was significantly higher, compared with the levels detected in the IR and NP-MSC groups $(\mathrm{P}<0.01)$. These results suggested hat hypoxic preconditioning upregulated the expression of HIF- $1 \alpha$ in the BMSCs.

\section{Discussion}

The transplantation of BMSCs exerts protective effects on SCIRI (6-8), however, the survival rate of transplanted 
Sham

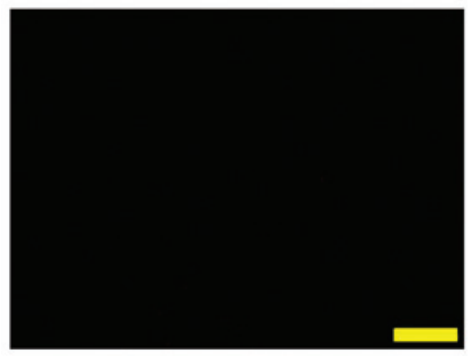

NP-MSCs

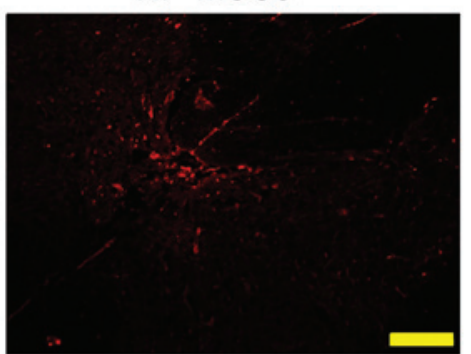

IR

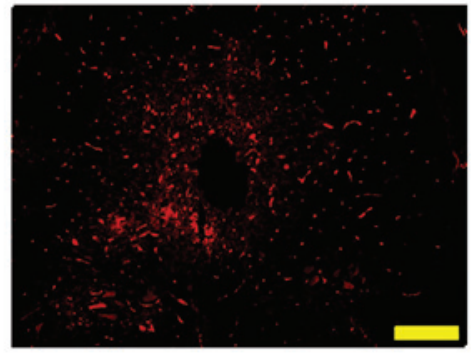

HP-MSCs

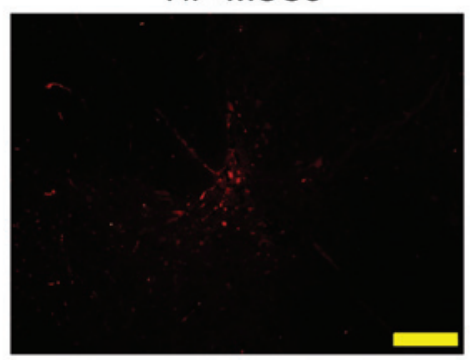

PBS
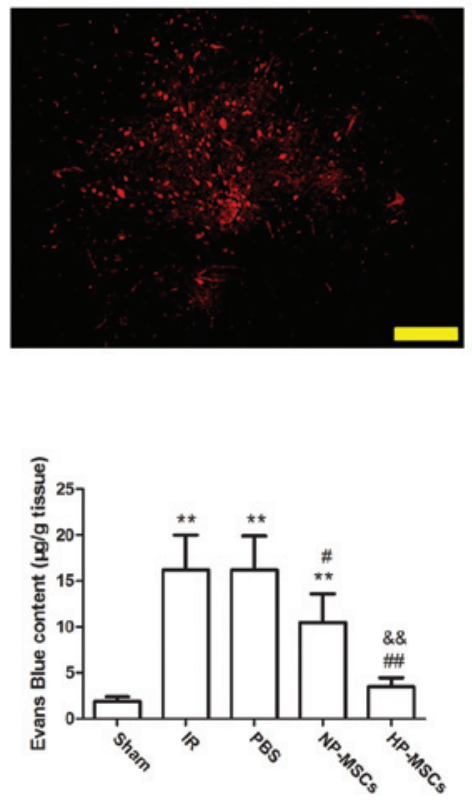

Figure 3. Detection of the integrity of the BSCB using Evans blue staining. The penetration of Evans blue (red) was observed under a fluorescence microscope (images are representative results from repeated experiments). The concentration of Evans blue were measured in the spinal cord tissue samples from each group. Each group contained six rats and the data are presented as the mean \pm standard deviation. Scale bar, $200 \mu \mathrm{m}$. ${ }^{* *} \mathrm{P}<0.01$, vs. sham group; ${ }^{\#} \mathrm{P}<0.05$ and ${ }^{\# \#} \mathrm{P}<0.01$, vs. IR group; and \&\& $\mathrm{P}<0.01$, vs. NP-MSC group. BSCB, blood spinal cord barrier; BMSC, bone marrow mesenchymal stem cell; Sham, sham-operated; IR, ischemia/reperfusion; NP-MSC, non-preconditioned BMSC transplantation; HP-MSC; hypoxic preconditioned BMSC transplantation; PBS, phosphate-buffered saline-treated.

\section{Sham}

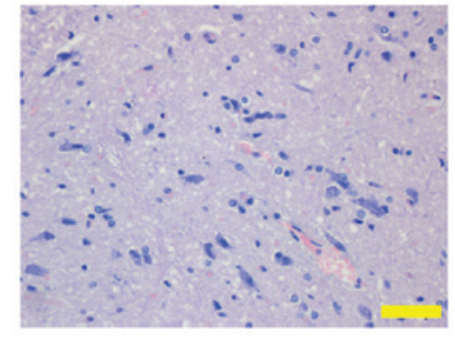

NP-MSCs

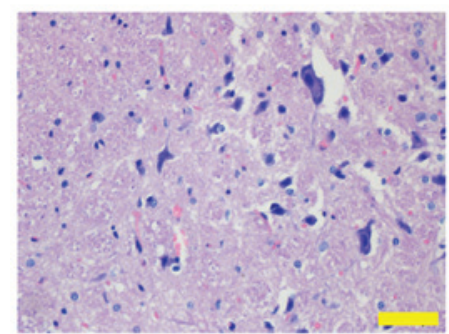

IR

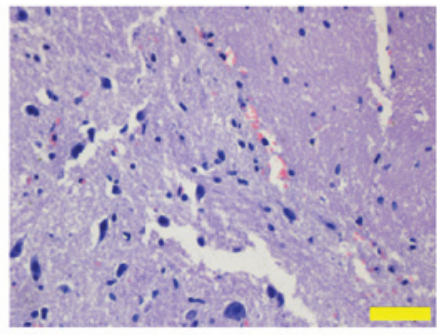

HP-MSCs

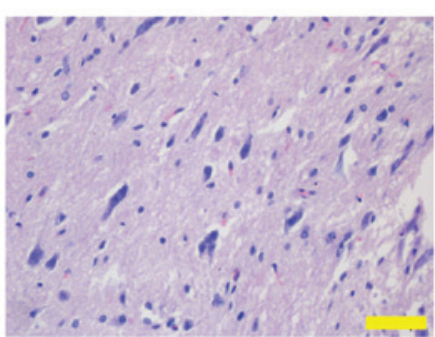

PBS
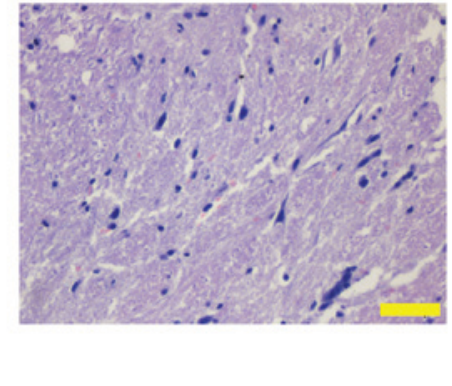

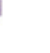


Sham

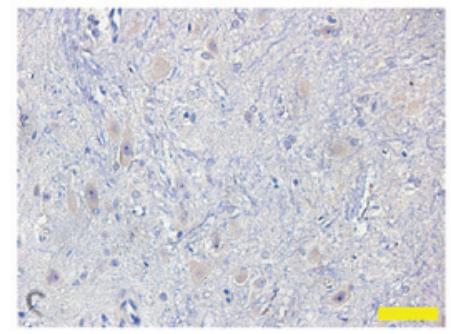

NP-MSCs

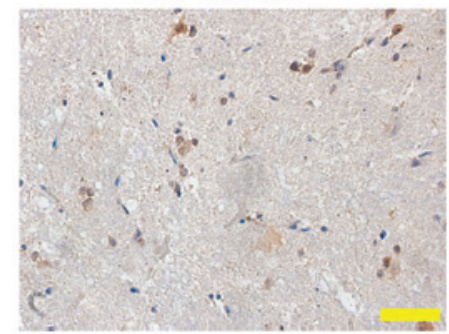

IR

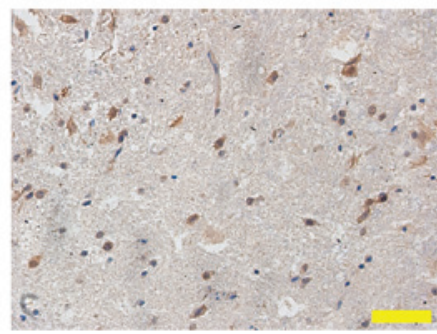

HP-MSCs

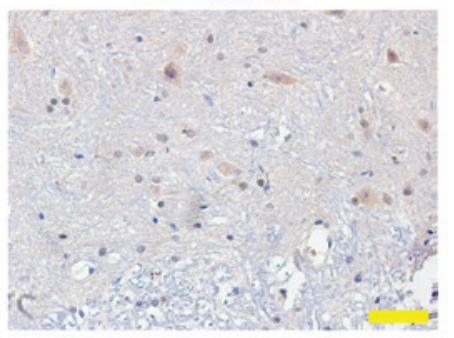

PBS

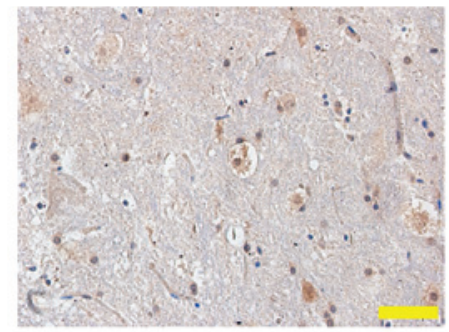

Figure 5. Detection of apoptosis in spinal cord tissue samples, determined using terminal deoxynucleotidyl transferase dUTP nick end labeling. Scale bar, $50 \mu \mathrm{m}$. BMSC, bone marrow stem cell; Sham, sham-operated; IR, ischemia/reperfusion; NP-MSC, non-preconditioned BMSC transplantation; HP-MSC; hypoxic preconditioned BMSC transplantation; PBS, phosphate-buffered saline-treated.

A

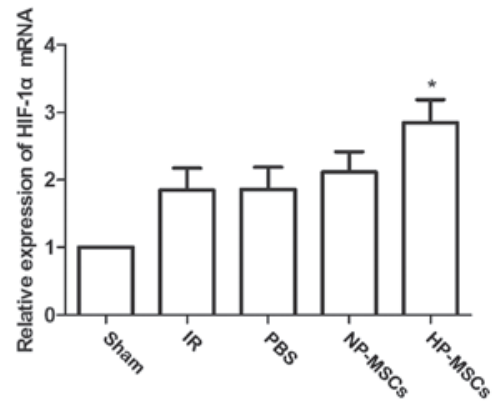

B
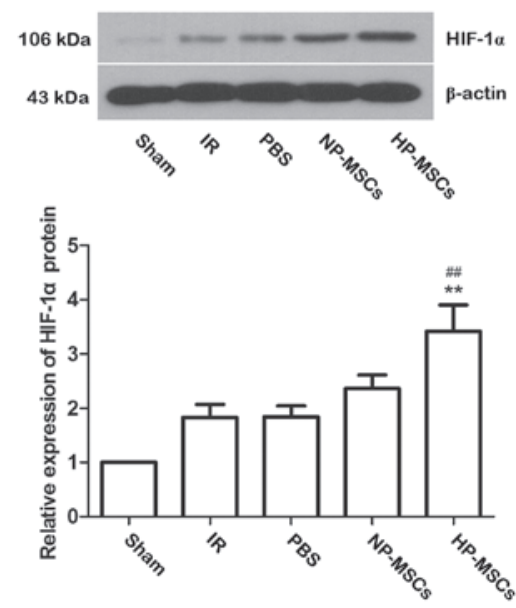

Figure 6. Expression of HIF-1 $\alpha$ in the spinal cord tissue samples of each group. (A) Detection of the mRNA expression levels of HIF-1 $\alpha$ using reverse transcription-quantitative polymerase chain reaction. (B) Detection of the protein expression levels of HIF-1 $\alpha$ by western blotting. Typical results of triplicate experiments are shown, with data presented as the mean \pm standard deviation. ${ }^{* *} \mathrm{P}<0.01$ and ${ }^{*} \mathrm{P}<0.05$, vs. IR group; ${ }^{\# \#} \mathrm{P}<0.01$, vs. NP-MSC group. BMSC, bone marrow stem cell; Sham, sham-operated; IR, ischemia/reperfusion; NP-MSC, non-preconditioned BMSC transplantation; HP-MSC; hypoxic preconditioned BMSC transplantation; PBS, phosphate-buffered saline-treated.
BMSCs secrete several cytokines, chemokines and growth factors, in order to promote tissue repair (24). Hypoxic conditions can promote the ability of BMSCs to secrete individual factors (25-27). Studies on cerebral ischemia/reperfusion injuries have demonstrated that hypoxic preconditioning increases the secretion levels of hepatocyte growth factor (HGF) and vascular endothelial growth factor (VEGF) by BMSCs, thus increasing cognitive function and promoting neurogenesis in rats $(16,28)$. The neurological function score of the HP-MSC group in the present study was significantly higher, compared with that of the NP-MSC group, indicating that hypoxic preconditioning increased the protective effects of BMSCs on neurological function; however, whether the increased protective effects were associated with the secretion of HGF and VEGF requires further detailed investigation.

The BSCB regulates and limits molecules entering the central nervous system and maintains a normal microenvironment in the spinal cord (29). Primary injury can immediately induce BSCB damage, and the damaged BSCB changes the permeability of proteins so that inflammatory substances can enter freely, thus inducing and aggravating spinal cord injury (30). Therefore, the destruction of the BSCB is one of the central links to SCIRI. The present study demonstrated that, compared with the NP-MSC group, the concentration of Evans blue in the spinal cord tissues samples of the HP-MSC group were significantly decreased, indicating that hypoxic preconditioning increased the protective effect of BMSCs on the BSCB. Notably, this mechanism was important in the reduction of secondary injury following SCIRI.

Hypoxic preconditioning increases the repair capacity of BMSCs in myocardial tissues and can promote angiogenesis (31); the ability of hypoxic preconditioned BMSCs to inhibit apoptosis in ischemic tissues is was also increased, compared with BMSCs without preconditioning $(32,33)$. The 
present study determined that the effects of hypoxic preconditioned BMSCs on the repair of spinal cord tissues following ischemia/reperfusion injury, and on the inhibition of apoptosis, were significantly increased, which further confirmed that hypoxic preconditioning increased the protective effect of BMSCs on SCIRI.

HIF- $1 \alpha$ is a nuclear transcription factor, the expression of which is upregulated under hypoxic conditions, which can increase the tolerance of tissues and cells to hypoxic environments (34). Several in vitro studies have revealed that hypoxic preconditioning promotes the secretion of HIF-1 $\alpha$ by BMSCs $(35,36)$. The present study determined that the expression levels of HIF-1 $\alpha$ in the spinal cord tissue samples of the HP-MSC group were significantly higher, compared with those of the NP-MSC group, indicating that hypoxic preconditioning increased the protective effect of BMSCs on SCIRI by promoting the expression of HIF-1 $\alpha$. These results were concordant with those of a previous study investigating renal ischemia/reperfusion injury (37).

HIF-1 $\alpha$ can induce gene expression and resume function of the cellular internal environment in the absence of oxygen $(38,39)$. The results of the present study suggested that hypoxic preconditioning unregulated the protein and mRNA expression levels of HIF-1 $\alpha$ in BMSCs, improving the function of motor nerve cells following SCIRI. It also inhibited apoptosis and protected the integrity of the BSCB, all of which can enhance the repair capacity of BMSCs on tissue injury. These functions may be associated with upregulation of the expression of HIF-1 $\alpha$ following hypoxic preconditioning. In conclusion, hypoxic preconditioning was found to be an effective means for increasing the survival rate of transplanted BMSCs, and for promoting the protective effects of BMSCs on SCIRI. The results suggest that hypoxic preconditioning may serve as a promising adjuvant therapeutic strategy for the treatment of SCIRI.

\section{Acknowledgements}

The present study was supported by grants from the National Natural Science Foundation of China (grant no. 81401000), the Science and Technology Foundation of the Liaoning Province (grant no. 2012408002), the Doctoral Research Foundation of the Liaoning Province (grant no. 20141035) and the Foundation for Scientific Research of The First Affiliated Hospital of the China Medical University (grant no. 2014-07).

\section{References}

1. Weir CJ, Zivin JA and Lyden PD: Inter-relationships between spinal cord blood flow, neuronal death and neurological function in rabbit spinal cord ischemia. Brain Res 946: 43-51, 2002.

2. Kuniyoshi Y, Koja K, Miyagi K, Shimoji M, Uezu T, Arakaki K, Yamashiro S, Mabuni K, Senaha S and Nakasone Y: Prevention of postoperative paraplegia during thoracoabdominal aortic surgery. Ann Thorac Surg 76: 1477-1484, 2003.

3. MacArthur RG, Carter SA, Coselli JS and LeMaire SA: Organ protection during thoracoabdominal aortic surgery: Rationale for a multimodality approach. Semin Cardiothorac Vasc Anesth 9: 143-149, 2005

4. Sinha AC and Cheung AT: Spinal cord protection and thoracic aortic surgery. Curr Opin Anaesthesiol 23: 95-102, 2010.
5. Mauney MC, Blackbourne LH, Langenburg SE, Buchanan SA, Kron IL and Tribble CG: Prevention of spinal cord injury after repair of the thoracic or thoracoabdominal aorta. Ann Thorac Surg 59: 245-252, 1995

6. Shi E, Kazui T, Jiang X, Washiyama N, Yamashita K, Terada H and Bashar AH: Intrathecal injection of bone marrow stromal cells attenuates neurologic injury after spinal cord ischemia. Ann Thorac Surg 81: 2227-2233; discussion 2233-2224, 2006.

7. Shi E, Kazui T, Jiang X, Washiyama N, Yamashita K, Terada H and Bashar AH: Therapeutic benefit of intrathecal injection of marrow stromal cells on ischemia-injured spinal cord. Ann Thorac Surg 83: 1484-1490, 2007.

8. Fang B, Wang H, Sun XJ, Li XQ, Ai CY, Tan WF, White PF and $\mathrm{Ma} \mathrm{H}$ : Intrathecal transplantation of bone marrow stromal cells attenuates blood-spinal cord barrier disruption induced by spinal cord ischemia-reperfusion injury in rabbits. J Vasc Surg 58: 1043-1052, 2013

9. Pagani FD, DerSimonian H, Zawadzka A, Wetzel K, Edge AS, Jacoby DB, Dinsmore JH, Wright S, Aretz TH, Eisen HJ and Aaronson KD: Autologous skeletal myoblasts transplanted to ischemia-damaged myocardium in humans. Histological analysis of cell survival and differentiation. J Am Coll Cardiol 41: 879-888, 2003

10. Toma C, Pittenger MF, Cahill KS, Byrne BJ and Kessler PD: Human mesenchymal stem cells differentiate to a cardiomyocyte phenotype in the adult murine heart. Circulation 105: 93-98, 2002.

11. Hodgetts SI, Beilharz MW, Scalzo AA and Grounds MD: Why do cultured transplanted myoblasts die in vivo? DNA quantification shows enhanced survival of donor male myoblasts in host mice depleted of CD4+ and CD8+ cells or Nk1.1+ cells. Cell transplant 9: 489-502, 2000.

12. Huang X, Su K, Zhou L, Shen G, Dong Q, Lou Y and Zheng S: Hypoxia preconditioning of mesenchymal stromal cells enhances PC 3 cell lymphatic metastasis accompanied by VEGFR-3/CCR7 activation. J Cell Biochem 114: 2834-2841, 2013.

13. Kim HW, Haider HK, Jiang S and Ashraf M: Ischemic preconditioning augments survival of stem cells via miR-210 expression by targeting caspase-8-associated protein 2 . J Biol Chem 284: 33161-33168, 2009.

14. Peterson KM, Aly A, Lerman A, Lerman LO and Rodriguez-Porcel M: Improved survival of mesenchymal stromal cell after hypoxia preconditioning: Role of oxidative stress. Life Sci 88: 65-73, 2011.

15. Liu H, Liu S, Li Y, Wang X, Xue W, Ge G and Luo X: The role of SDF-1-CXCR4/CXCR7 axis in the therapeutic effects of hypoxia-preconditioned mesenchymal stem cells for renal ischemia/reperfusion injury. PloS one 7: e34608, 2012.

16. Chang $\mathrm{CP}$, Chio $\mathrm{CC}$, Cheong $\mathrm{CU}$, Chao $\mathrm{CM}$, Cheng $\mathrm{BC}$ and Lin MT: Hypoxic preconditioning enhances the therapeutic potential of the secretome from cultured human mesenchymal stem cells in experimental traumatic brain injury. Clin Sci (Lond) 124: 165-176, 2013.

17. Carr CA, Stuckey DJ, Tatton L, Tyler DJ, Hale SJ, Sweeney D, Schneider JE, Martin-Rendon E, Radda GK, Harding SE, et al: Bone marrow-derived stromal cells home to and remain in the infarcted rat heart but fail to improve function: An in vivo cine-MRI study. Am J Physiol Heart Circ Physiol 295: H533-H542, 2008.

18. Yu J, Yin S, Zhang W, Gao F, Liu Y, Chen Z, Zhang M, He J and Zheng S: Hypoxia preconditioned bone marrow mesenchymal stem cells promote liver regeneration in a rat massive hepatectomy model. Stem Cell Res Ther 4: 83, 2013.

19. Lang-Lazdunski L, Heurteaux C, Mignon A, Mantz J, Widmann C, Desmonts J and Lazdunski M: Ischemic spinal cord injury induced by aortic cross-clamping: Prevention by riluzole. Eur J Cardiothorac Surg 18: 174-181, 2000.

20. Huang Y, Xie K, Li J, Xu N, Gong G, Wang G, Yu Y, Dong H and Xiong L: Beneficial effects of hydrogen gas against spinal cord ischemia-reperfusion injury in rabbits. Brain Res 1378: 125-136, 2011.

21. Wang Q, Ding Q, Zhou Y, Gou X, Hou L, Chen S, Zhu Z and Xiong L: Ethyl pyruvate attenuates spinal cord ischemic injury with a wide therapeutic window through inhibiting high-mobility group box 1 release in rabbits. Anesthesiology 110: 1279-1286, 2009.

22. Livak KJ and Schmittgen TD: Analysis of relative gene expression data using real-time quantitative PCR and the 2(-Delta Delta C(T)) Method. Methods 25: 402-408, 2001 
23. De Ugarte DA, Alfonso Z, Zuk PA, Elbarbary A, Zhu M Ashjian P, Benhaim P, Hedrick MH and Fraser JK: Differential expression of stem cell mobilization-associated molecules on multi-lineage cells from adipose tissue and bone marrow. Immunol Lett 89: 267-270, 2003.

24. Caplan AI and Dennis JE: Mesenchymal stem cells as trophic mediators. J Cell Biochem 98: 1076-1084, 2006.

25. Kinnaird T, Stabile E, Burnett MS, Lee CW, Barr S, Fuchs S and Epstein SE: Marrow-derived stromal cells express genes encoding a broad spectrum of arteriogenic cytokines and promote in vitro and in vivo arteriogenesis through paracrine mechanisms. Circ Res 94: 678-685, 2004.

26. Das R, Jahr H, van Osch GJ and Farrell E: The role of hypoxia in bone marrow-derived mesenchymal stem cells: Considerations for regenerative medicine approaches. Tissue Eng Part B Rev 16: 159-168, 2010.

27. Rosová I, Dao M, Capoccia B, Link D and Nolta JA: Hypoxic preconditioning results in increased motility and improved therapeutic potential of human mesenchymal stem cells. Stem Cells 26: 2173-2182, 2008.

28. Bernaudin M, Tang Y, Reilly M, Petit E and Sharp FR: Brain genomic response following hypoxia and re-oxygenation in the neonatal rat. Identification of genes that might contribute to hypoxia-induced ischemic tolerance. J Biol Chem 277: 39728-39738, 2002.

29. Pan W and Kastin AJ: Cytokine transport across the injured blood-spinal cord barrier. Curr Pharm Des 14: 1620-1624, 2008.

30. Profyris C, Cheema SS, Zang D, Azari MF, Boyle K and Petratos S: Degenerative and regenerative mechanisms governing spinal cord injury. Neurobiol Dis 15: 415-436, 2004.
31. Wang JA, He A, Hu X, Jiang Y, Sun Y, Jiang J, Gui C, Wang Y and Chen $\mathrm{H}$ : Anoxic preconditioning: A way to enhance the cardioprotection of mesenchymal stem cells. Int J Cardiol 133: 410-412, 2009.

32. Li JH, Zhang $\mathrm{N}$ and Wang JA: Improved anti-apoptotic and anti-remodeling potency of bone marrow mesenchymal stem cells by anoxic pre-conditioning in diabetic cardiomyopathy. J Endocrinol Invest 31: 103-110, 2008.

33. He A, Jiang Y, Gui C, Sun Y, Li J and Wang JA: The antiapoptotic effect of mesenchymal stem cell transplantation on ischemic myocardium is enhanced by anoxic preconditioning. Can J Cardiol 25: 353-358, 2009.

34. Majmundar AJ, Wong WJ and Simon MC: Hypoxia-inducible factors and the response to hypoxic stress. Mol Cell 40: 294-309, 2010.

35. Kanichai M, Ferguson D, Prendergast PJ and Campbell VA Hypoxia promotes chondrogenesis in rat mesenchymal stem cells: A role for AKT and hypoxia-inducible factor (HIF)-1alpha. J Cell Physiol 216: 708-715, 2008.

36. Liu H, Xue W, Ge G, Luo X, Li Y, Xiang H, Ding X, Tian P and Tian X: Hypoxic preconditioning advances CXCR4 and CXCR7 expression by activating HIF-1alpha in MSCs. Biochem Biophys Res Commun 401: 509-515, 2010.

37. Ma D, Lim T, Xu J, Tang H, Wan Y, Zhao H, Hossain M, Maxwell PH and Maze M: Xenon preconditioning protects against renal ischemic-reperfusion injury via HIF-lalpha activation. J Am Soc Nephrol 20: 713-720, 2009.

38. Singh N, Sharma G and Mishra V: Hypoxia inducible factor-1: Its potential role in cerebral ischemia. Cell Mol Neurobiol 32: 491-507, 2012.

39. Hirota K: Hypoxia-inducible factor 1, a master transcription factor of cellular hypoxic gene expression. J Anesth 16: 150-159, 2002. 